Lara N. Schulze*\#1, Sandra Van der Auwera\#1,2, Deborah Janowitz' ${ }^{1}$ Johannes Hertel'1, Katharina Wittfeld ${ }^{112}$, René Walk', Nele Friedrich ${ }^{3,4}$, Henry Völzke ${ }^{3,5}$, Hans J. Grabe ${ }^{1,2}$

\title{
The Impact of Childhood Trauma and Depressive Symptoms on Body Mass Index
}

\author{
'Department of Psychiatry and Psychotherapy, University Medicine Greifswald, Germany \\ ${ }^{2}$ German Center for Neurodegenerative Diseases (DZNE), Site Rostock/ Greifswald, Germany \\ ${ }^{3}$ Institute of Clinical Chemistry and Laboratory Medicine, University Medicine Greifswald, Germany \\ ${ }^{4}$ German Center for Cardiovascular Research (DZHK), partner site Greifswald \\ 5 Institute for Community Medicine, University Medicine Greifswald, Greifswald, Germany \\ *email: lara.strobelduni-greifswald.de \\ \# Lara N. Schulze und Sandra Van der Auwera are joint first authors contributed equally.
}

DOI: 10.2478/gp-2019-0008

Received: 6 March 2019; Accepted: 21 March 2019

\begin{abstract}
Objectives: Obesity is associated with several somatic diseases and increased psychological burden. This study focused on two potential psychological predictors of the body mass index (BMI), childhood trauma and depressive symptoms. Methods: We used three independent populations: two general population samples (Study of Health in Pomerania, SHIP-2, N = 1,657; SHIP-TREND-0, N $=3,278$ ) and one patient sample (GANI_MED, $N=1,742)$. Childhood trauma was measured with the childhood trauma questionnaire (CTQ) and depression with the Beck Depression Inventory (BDI-II) in SHIP-2 and the Patient Health Questionnaire (PHQ-9) in SHIP-TREND-0 and GANI_MED. We investigated the impact of childhood trauma and depression on BMI. Furthermore, we used mediation analysis to assess whether depression was a significant mediator on the path from childhood trauma to adult BMI in each of the three samples. Results: In all the three populations, depressive symptoms exhibited a significant association towards higher BMI $(p<0.05)$. Childhood trauma was positively associated with BMI with significant associations in SHIP-TREND-0 ( $p$ $<0.001)$ and GANI_MED ( $p=0.005)$. The relationship between CTQ and BMI was significantly partially mediated $(p<0.05)$ by depressive symptoms in SHIP-TREND-0 (38.0\%) and GANI_MED (16.4\%), in SHIP-2 results pointed in the same direction. All the trauma sub-dimensions, except sexual abuse, exhibited at least one significant association towards increased BMI in one of the samples. Conclusions: Childhood trauma and depressive symptoms may be considered as causes of obesity. These results suggest that psychological treatments against obesity should address childhood maltreatment as well as depressive symptoms in their diagnostic assessment and could facilitate psychotherapeutic treatment when necessary.
\end{abstract}

\section{Keywords}

Depression, trauma, obesity, epidemiology, live events

\section{INTRODUCTION}

One-third of the U.S. population (Ogden et al. 2014) and onefourth of the German population (Völzke et al. 2015) have a strikingly increased body mass index $(\mathrm{BMI}>30)$. This is a serious public and individual health problem since obesity is associated with several somatic diseases and increased psychological burden (Janowitz et al. 2014; McCarthy-Jones and McCarthy-Jones 2014). Our study will be focused on two potential psychological predictors of overweight, childhood trauma and depressive symptoms.

\section{Childhood trauma and BMI}

Childhood trauma can be classified in different categories: Emotional abuse refers to verbal aggression, humiliation or degradation by an adult person. Physical aggression or violation stands for physical abuse. Sexual abuse is defined as sexual contact between the child and an older person. Besides these three categories of abuse, two further categories of childhood neglect exist: 'Emotional neglect refers to the failure of caretakers to provide a child's basic psychological and emotional needs [...]' (Bernstein and Fink 1998). The failure of caregivers to provide the child's basic physical needs (e.g., food, supervision) is defined as physical neglect. Regarding the 
variability of childhood maltreatment in general population, more people report on at least mild forms of emotional (40.7\%) or physical neglect $(41.7 \%)$ than on emotional (11.9\%), physical (9.1\%) or sexual (6.6\%) abuse (Schulz et al. 2014).

The association between childhood trauma and higher BMI values has been investigated in several studies. In a prospective assessment, the long-term impact of childhood trauma on obesity was demonstrated (Bentley and Widom 2009): The authors differentiated between sexual and physical abuse and neglect. In a sample of almost 700 participants, physical abuse predicted higher BMI values whereas neglect did not. In adolescence, exposure to violence or witnessing of physical abuse or domestic violence was also related to a significantly higher risk of obesity (Gooding et al. 2015). Another study suggest that only in women, sexual abuse was associated with higher BMI values (Mamun 2007). These studies provide evidence for childhood trauma as a well-founded factor in the aetiology of obesity. However, it still remains unclear which sub-dimensions of childhood adversity are linked to high body weight.

\section{Depressive symptoms and BMI}

Recurrent depressive disorder has been repeatedly associated with higher BMI values (Farmer et al. 2008). A meta-analysis of 16 studies resumed, that depressed compared to non-depressed subjects have a higher risk to become obese (Blaine 2008). Moreover, the association between depression and higher BMI values was stronger in females than in males and the risk was particularly higher for adolescent females (Blaine 2008). In a prospective examination, the role of potential mediators (depression, anxiety, posttraumatic stress and trauma) was analysed to explain the relationship between childhood trauma and obesity (Francis et al. 2015). 78 participants with childhood trauma and 349 non-maltreated participants were investigated over a period of 16 years for three follow-ups. The authors concluded that depression leads to obesity and anxiety mediates the relationship between physical abuse and higher BMI values in women. Furthermore childhood trauma has consistently been associated with the onset of depressive symptoms in adults (Boynton-Jarrett et al. 2012; Schulz et al. 2014).

\section{Hypotheses}

In sum, the previous investigations support the hypothesis that both depression and childhood traumatization are associated with obesity. However, it is still unclear whether childhood trauma and depressive disorders are independent in their effects on body weight or whether adult depression mediates the effects of childhood trauma on body weight as studies with large sample sizes and replication samples are still missing to date. Moreover, the putative role of childhood trauma subtypes is unknown.

By testing the association between childhood trauma, depression and BMI in three independent large samples, we validate prior results and offer a generalization of the findings, as two studies are based on general population samples (SHIP2, SHIP-TREND-0) and one study is a large clinical sample (GANI_MED). We hypothesize that: 1. Childhood trauma is associated with increased BMI and depression score in adulthood; 2. Current depression and BMI in adulthood are associated; 3. Part of the effect from childhood trauma to BMI is mediated by current depressive symptoms.

\section{MATERIALS AND METHODS}

\section{The Study of Health in Pomerania}

Data from the 'Study of Health in Pomerania (SHIP)' were used (Völzke et al. 2011).

SHIP-2: The target population was comprised of adult German residents in northeast Germany living in 3 cities and 29 communities, with a total population of 212,157 . A twostage stratified cluster sample of adults aged 20 to 83 years was drawn from the local population registration files. The net sample comprised 6,267 eligible subjects, of which 4,308 Caucasian subjects participated at baseline SHIP-0 between 1997 and 2001. Follow-up examination (SHIP-1) was conducted 5 years after the baseline and included 3,300 subjects. From 2008 to 2012, the third phase of data collection (SHIP-2, N = 2,333 ) was carried out. In parallel, a psychiatric follow-up, the SHIP-LEGEND study ('Life-Events and Gene-Environment Interaction in Depression'), was conducted. Among the 3,669 subjects of SHIP-0 that were invited to take part in the SHIPLEGEND study, 2,400 participated.

SHIP-TREND-0: In 2008, an independent sample called SHIPTREND-0 $(\mathrm{N}=4,420)$ from the same area was drawn and similar examinations like in SHIP-0 were undertaken. The objective was to compare two samples from the same target population but from different time periods (1997 and 2008) concerning disease prevalence and risk behaviour.

\section{GANI_MED}

From July 2011, patients for the 'Greifswald Approach to Individualized Medicine' (GANI_MED) were recruited at the University Medicine Greifswald (Grabe et al., 2014) The 
GANI_MED patients $(\mathrm{N}=3872)$ belong to six main cohorts with common cardiovascular, cerebrovascular or metabolic conditions: heart failure $(\mathrm{N}=1078)$, stroke $(\mathrm{N}=430)$, periodontal disease $(\mathrm{N}=661)$, renal insufficiency $(\mathrm{N}=392)$, metabolic syndrome $(\mathrm{N}=879)$, and fatty liver disease $(\mathrm{N}=$ 270 ) and three additional cohorts (patients with sepsis [ $\mathrm{N}=25]$, pulmonary diseases $[\mathrm{N}=137]$ ), and adverse medication effects.

\section{Phenotype Measures}

Sociodemographic factors and medical history were assessed by a computer-assisted face-to-face interview. The following information was used: Smoking status (never-smoker, exsmoker and current smoker), height (to the nearest $1.0 \mathrm{~cm}$ ) and weight (to the nearest $0.1 \mathrm{~kg}$ with the subject in light clothing and without shoes). From these measurements, the BMI of each subject was calculated $\left(\mathrm{BMI}=(\right.$ weight in $\left.\mathrm{kg}) /(\text { height in } \mathrm{m})^{2}\right)$.

Current depressive symptoms were assessed in SHIP-LEGEND using the Beck Depression Inventory (BDI-II), which is a 21-item self-report questionnaire with high reliability $(0.89-$ 0.93) and validity (Herzberg et al. 2008). In SHIP-TREND-0 and GANI_MED, the Patient Health Questionnaire (PHQ-9) was used, a 9-item self-report questionnaire also with high reliability (.86-.89) and validity (Hawley et al. 2013; Kroenke et al. 2001).

In SHIP-LEGEND and SHIP-TREND-0, the Childhood Trauma Questionnaire (CTQ) was used for self-report of childhood maltreatment. This questionnaire asks for longlasting type-II-traumata of five different categories: emotional, physical and sexual abuse as well as emotional and physical neglect (Bernstein and Fink 1998). The CTQ has 34 items that were rated on a five-point Likert scale with higher scores indicating more self-rated exposure to traumatic events. The manual provides dimensional scores to determine the severity of abuse and neglect. To investigate the role of an increasing severity of childhood trauma on BMI, we calculated the overall sum score of childhood trauma as well as the sub-scores for the three abuse and two neglect dimensions. In GANI_MED, the CTQ screener (CTS) (Glaesmer et al. 2013; Grabe et al. 2012) was used with one question for each abuse and neglect dimension rated on a five-point Likert scale with higher scores indicating more self-rated exposure to traumatic events. Similar to SHIP2 and SHIP-TREND-0, we calculated an overall trauma score as the sum of all sub-dimensions. For the single dimension analysis, we used the raw response to each of the five questions.

From statistical analysis, we excluded all the subjects with missing data (overlap exists) for BMI (SHIP-2 $\mathrm{N}=6$, SHIP-
TREND-0 N = 7, GANI_MED $\mathrm{N}=$ 201), CTQ (SHIP-2 N $=512$, SHIP-TREND-0 $\mathrm{N}=806$, GANI_MED $\mathrm{N}=1596$ ) variables, PHQ-9/BDI-II (SHIP-2 $\mathrm{N}=$ 491, SHIP-TREND-0 $\mathrm{N}=748$, GANI_MED $\mathrm{N}=1714$ ) and smoking (SHIP-2 $\mathrm{N}=$ 6, SHIP-TREND-0 $\mathrm{N}=22$, GANI_MED $\mathrm{N}=106$ ). We also excluded pregnant woman (SHIP-2 $\mathrm{N}=2$, SHIP-TREND-0 N $=9$, GANI_MED N = 103 from gynaecology) and underweight people (SHIP-2 $\mathrm{N}=149$, SHIP-TREND-0 N = 236, GANI_ MED $N=197$ ) due to the BMI thresholds for underweight recommended by the $\mathrm{WHO}$, since eating disorders like anorexia nervosa or bulimia nervosa are assumed. For this clinical relevant subpopulation, higher rates of childhood abuse are also reported (Racine and Wildes 2015; Rorty et al. 1994). To compare the effect estimates for childhood trauma scores and depression scores measured with different scales, variables were $\mathrm{z}$-standardized ( $\mathrm{z}$-transformation, $\mathrm{z}=(\mathrm{x}$-mean $(\mathrm{x})) / \mathrm{sd}(\mathrm{x}))$ before analysis (Milligan und Cooper 1988).

\section{Statistical Analysis}

1. Linear regression analyses were applied to investigate the association between the CTQ measurements (continuous CTQ sum score and five dimensional subscores) with BDI-II/PHQ-9 and BMI. As all the three continuous measurements were not normally distributed, bootstrapping with 2000 replications was applied (Harrell 2001). Bootstrapping is a non-parametric resampling method where the same statistic is calculated several times in the same sample to determine a confidence interval for the statistical effect.

2. Linear regression analyses were applied to investigate the association between BDI-II/PHQ-9 and BMI as outcome. As all the three continuous measurements were not normally distributed, bootstrapping with 2000 replications was applied.

3. To assess a potentially mediating effect of the current depression on the path from CTQ to BMI, we used the medeff function of the STATA mediation package for continuous outcome variable and mediator. In mediation analysis, we aim to explain the relationship between exposure and outcome variable by introducing a third variable, the mediator. As a precondition to perform mediation analysis, this mediator variable is associated with the exposure as well as with the outcome (Vittinghoff et al. 2012)

All the analyses were controlled for age, sex and smoking status. Statistical analyses were performed using STATA/MP software, version 13 . 


\section{RESULTS}

The final study sample comprised $\mathrm{N}=1,657$ (52\% females) subjects for SHIP-2, $\mathrm{N}=3,278$ ( $50 \%$ females) for SHIPTREND- 0 and N = 1,742 (41\% females) for GANI_MED. Mean age was $57 \pm 13,51 \pm 15$ and $59 \pm 15$ years for SHIP-2, SHIPTREND-0 and GANI_MED, respectively. Detailed sample characteristics on the sociodemographic and psychometric variables are given in Table 1 . In all the three cohorts, the highest prevalence of childhood traumatization was reported for both forms of neglect, whereas the lowest prevalence could be found for sexual abuse (Table 1).

Hypothesis 1: In SHIP-2, no significant effect of a higher childhood trauma score (CTQ) on BMI was found, although the effect pointed to a positive association between higher load of childhood trauma and BMI $(\beta=0.16, \mathrm{p}=0.14)$ (Table 2$)$. However, a significant positive association was found between physical neglect and BMI $(\beta=0.26, \mathrm{p}=0.028)$ (Table 2$)$.

For SHIP-TREND-0, the association between CTQ score and BMI was significantly supported $(\beta=0.36, \mathrm{p}<0.001)$. All the CTQ sub-dimensions, except sexual abuse, showed a similar positive association to BMI with the strongest associations for emotional $(\beta=0.37, \mathrm{p}<0.001)$ and physical abuse $(\beta=0.31, \mathrm{p}=$ $0.003)$ as well as physical neglect $(\beta=0.31, \mathrm{p}=0.001)$.

Our clinical sample, GANI_MED, also confirmed the positive association between CTQ and BMI $(\beta=0.42, p=0.005)$. Of the sub-dimensions, the strongest associations were found for physical abuse $(\beta=0.43, p=0.006)$ and emotional neglect $(\beta=$ $0.37, \mathrm{p}=0.005)$.

In sum, all the sub-dimensions, except sexual abuse, exhibit at least one significant association towards increased BMI in one of the three samples.

Hypothesis 2: The depression scores (BDI-II and PHQ-9) showed significant associations towards BMI in all the three samples (SHIP-2: $\beta=0.30, p=0.027$; SHIP-TREND-0: $\beta=0.56$, $\mathrm{p}<0.001$; GANI_MED: $\beta=0.34, \mathrm{p}=0.038$ ). These effects were even stronger than the effects of the CTQ score in SHIP-2 and SHIP-TREND-0 (Table 2).

Hypothesis 3: As depressive symptoms were associated with CTQ (data not shown) and BMI, requirements for a mediator were fulfilled. The effect estimates for the CTQ score on BMI decreased for all three cohorts, when additionally adjusting for the depression score. That part of the CTQ main effect now being explained on the path through depressive symptoms is the mediation effect (figure 1). Although the CTQ failed to be predictive for BMI in SHIP-2, we observed a partial mediation effect through depressive symptoms that slightly missed significance (average mediated effect 45\%). In SHIP-TREND-0, a significant $(\mathrm{p}<0.05)$ partial mediation was observed: $38 \%$ of the direct effect from CTQ score to BMI were mediated via depression score. In GANI_MED, the mediated effect (16.4\%, $\mathrm{CI}=0.003-016)$ was significant $(\mathrm{p}<0.05)$ too.

All the analyses were conducted gender stratified, but no appreciable differences were found and effects pointed always in the same direction (data not shown).

\section{DISCUSSION}

We did generally support the hypothesis on associations between childhood trauma as well as depressive symptoms and BMI in three independent large samples. These results underline former assessments, which postulated the impact of childhood trauma on obesity (McCarthy-Jones and McCarthyJones 2014; Bentley and Widom 2009, Gooding et al. 2015; Francis et al. 2015).

\section{Childhood trauma and BMI}

The non-significance in SHIP-2 could be explained by the fact, that all the participants are 30 years or older; so an important age group (20-29 years) was missed in this sample. Especially in adolescence, a strong association between childhood maltreatment and obesity has been observed (Gooding et al. 2015). The missing age group in SHIP-2 could lead to a reduced association strength. Interpreting the observed effect of childhood trauma on higher BMI, it is important to mention, that the CTQ asks for long-lasting type-II-traumata leading to persisting chronic stress (Bornstein et al. 2006). A possible way of stress reduction could be an affect regulation by eating leading to higher BMI values. Another explanation for the association between childhood trauma and higher BMI values is a limited feeling of satiety due to an elevated stress system leading to an enhanced food intake. Concerning the sub-dimensions, it is shown that the observed associations were influenced by an overall trauma load rather than triggered by individual trauma sub-dimensions. Physical and emotional neglect were the most prevalent childhood trauma dimensions in all three cohorts, whereas sexual abuse was least prevalent. For sexual abuse this lack of association could be due to the small variance in sexual abuse and the low number of sexual abuse cases. These results underline, that even physical and emotional neglect are strong 
Table 1. Descriptive statistics of all the three study samples: SHIP-2, SHIP-TREND-0 and GANI_MED.

\begin{tabular}{|c|c|c|c|}
\hline & SHIP-2 (N = 1657) & SHIP-TREND-O (N = 3278) & GANI_MED ( $N=1742)$ \\
\hline $\begin{array}{c}\text { Sex } \\
\text { females }\end{array}$ & $867(52 \%)$ & $1651(50 \%)$ & $717(41 \%)$ \\
\hline Age (mean $\pm s d$ ) & $57 \pm 13$ (range: $31-93$ ) & $51 \pm 15$ (range: $20-82$ ) & $59 \pm 48$ (range: $19-92$ ) \\
\hline $\begin{array}{l}\text { Smoking status } \\
\text { never } \\
\text { ex } \\
\text { current }\end{array}$ & $\begin{array}{l}639(39.6 \%) \\
688(41.5 \%) \\
330(19.9 \%)\end{array}$ & $\begin{array}{l}1221(37.3 \%) \\
1216(37.1 \%) \\
841(25.7 \%)\end{array}$ & $\begin{array}{c}801(46 \%) \\
597(34.3 \%) \\
344(19.8 \%)\end{array}$ \\
\hline $\mathrm{BMI}$ (mean $\pm \mathrm{sd}$ ) & $28.7 \pm 4.7$ (range: $19.7-49.4$ ) & $28.4 \pm 5.0$ (range: $18.5-54.4$ ) & $29.9 \pm 5.2$ (range: $18.9-59.1$ ) \\
\hline $\begin{array}{l}\text { Depression score (mean } \\
\qquad \pm \mathrm{sd} \text { ) }\end{array}$ & $\begin{array}{c}\text { BDI-II } \\
6.1 \pm 7 \text { (range: } 0-58 \text { ) }\end{array}$ & $\begin{array}{c}\text { PHQ-9 } \\
12.9 \pm 3.6 \text { (range: } 9-35 \text { ) }\end{array}$ & $\begin{array}{c}\text { PHQ-9 } \\
15.0 \pm 5.6 \text { (range: } 9-36 \text { ) }\end{array}$ \\
\hline $\begin{array}{c}\text { CTQ sum score (mean } \\
\pm \mathrm{sd} \text { ) }\end{array}$ & $33.7 \pm 9.4$ (range: $25-119$ ) & $33.1 \pm 9.5$ (range: $25-115$ ) & $8.0 \pm 3.0$ (range: $5-25$ ) \\
\hline Emotional abuse & $\begin{array}{c}6.2 \pm 2.4 \text { (range: } 5-25 \text { ) } \\
57.7 \% \text { no emotional abuse }\end{array}$ & $\begin{array}{c}6.2 \pm 2.4 \text { (range: } 5-25 \text { ) } \\
60.7 \% \text { no emotional abuse }\end{array}$ & $\begin{array}{c}1.3 \pm 0.9 \text { (range: } 1-5 \text { ) } \\
83.5 \% \text { no emotional abuse }\end{array}$ \\
\hline Physical abuse & $\begin{array}{c}5.8 \pm 1.9 \text { (range: } 5-25 \text { ) } \\
69.8 \% \text { no physical abuse }\end{array}$ & $\begin{array}{c}5.7 \pm 1.9 \text { (range: } 5-25 \text { ) } \\
74.4 \% \text { no physical abuse }\end{array}$ & $\begin{array}{c}1.4 \pm 0.8 \text { (range: } 1-5 \text { ) } \\
79.9 \% \text { no physical abuse }\end{array}$ \\
\hline Sexual abuse & $\begin{array}{l}5.2 \pm 1.3 \text { (range: } 5-25 \text { ) } \\
93,4 \% \text { no sexual abuse }\end{array}$ & $\begin{array}{l}5.2 \pm 1.3 \text { (range: } 5-25 \text { ) } \\
94,7 \% \text { no sexual abuse }\end{array}$ & $\begin{array}{c}1.1 \pm 0.4 \text { (range: } 1-5 \text { ) } \\
94,9 \% \text { no sexual abuse }\end{array}$ \\
\hline Emotional neglect & $9.2 \pm 4.4$ (range: $5-25$ ) & $9.0 \pm 4.3$ (range: $5-25$ ) & $1.9 \pm 1.1$ (range: $1-5$ ) \\
\hline Physical neglect & $\begin{array}{c}23.1 \% \text { no emotional neglect } \\
7.2 \pm 2.5 \text { (range: } 5-25 \text { ) } \\
37.9 \% \text { no physical neglect }\end{array}$ & $\begin{array}{c}25.8 \% \text { no emotional neglect } \\
7.0 \pm 2.5 \text { (range: } 5-25 \text { ) } \\
41.1 \% \text { no physical neglect }\end{array}$ & $\begin{array}{c}44.6 \% \text { no emotional neglect } \\
2.3 \pm 1.3 \text { (range: } 1-5 \text { ) } \\
39.6 \% \text { no physical neglect }\end{array}$ \\
\hline Clinical cohorts & NA & NA & $\begin{array}{c}\text { Renal Insufficiency: } 113 \text { (6.49\%) } \\
\text { Stroke: } 254 \text { (14.58\%) } \\
\text { Metabolic Syndrome: } \\
\text { - Cardiology: } 232(13.32 \%) \\
\text { - Psychiatry: } 163(9.36 \%) \\
\text { Fatty liver: } 126(7.23 \%) \\
\text { Periodontal disease: } 404(23.19 \%) \\
\text { Heart failure: } 393(22.56 \%) \\
\text { COPD: } 57(3.27 \%)\end{array}$ \\
\hline
\end{tabular}

BMI: Body Mass Index, CTQ: Childhood Trauma Questionnaire, COPD: Chronic Obstructive Pulmonary Disease, CVD: Cardiovascular Diseases

predictors for BMI. This is important to mention since more than $40 \%$ of the general population have reported at least mild forms of neglect (Schulz et al. 2014).

\section{Depressive symptoms and BMI}

The association between depressive symptoms and BMI was stronger in SHIP-2 and SHIP-TREND-0 than the association between childhood trauma and BMI, whereas in GANI_MED, the direct effect of childhood trauma on BMI was stronger. This suggests that in general population, depressive symptoms have a stronger effect on behaviour (e.g., activity and eating) than traumatization, whereas in the clinical population, childhood traumatization has a higher influence on BMI than current depressive symptoms. 
Table 2. Associations between CTQ variables, depression scores (standardized coefficients) and BMI in SHIP-2, SHIP-TREND-0 and GANI_ MED.

\begin{tabular}{|c|c|c|c|}
\hline & SHIP-2 (N=1657) & SHIP-TREND-0 ( $\mathrm{N}=3278)$ & GANI_MED (N=1742) \\
\hline \multicolumn{4}{|c|}{ Direct effect of СТQ variables on BMI * } \\
\hline CTQ sum score & $B=0.16 \pm 0.11, p=0.14$ & $B=0.36 \pm 0.1, p<0.001$ & $B=0.42 \pm 0.15, p=0.005$ \\
\hline Emotional abuse score & $B=0.09 \pm 0.12, p=0.45$ & $B=0.37 \pm 0.11, p<0.001$ & $B=0.27 \pm 0.16, p=0.087$ \\
\hline Physical abuse score & $B=0.09 \pm 0.12, p=0.45$ & $B=0.31 \pm 0.11, p=0.003$ & $B=0.43 \pm 0.16, p=0.006$ \\
\hline Sexual abuse score & $B=-0.07 \pm 0.12, p=0.56$ & $B=0.06 \pm 0.08, p=0.45$ & $B=0.30 \pm 0.21, p=0.15$ \\
\hline Emotional neglect score & $B=0.14 \pm 0.11, p=0.20$ & $B=0.27 \pm 0.09, p=0.003$ & $B=0.37 \pm 0.13, p=0.005$ \\
\hline Physical neglect score & $B=0.26 \pm 0.12, p=0.028$ & $B=0.31 \pm 0.09, p=0.001$ & $B=0.10 \pm 0.13, p=0.42$ \\
\hline \multicolumn{4}{|c|}{ Direct effect of BDI-II / PHQ-9 on BMI* } \\
\hline BDI-II/PHQ-9 & $B=0.3 \pm 0.14, p=0.027$ & $B=0.56 \pm 0.1, p<0.001$ & $B=0.34 \pm 0.16, p=0.038$ \\
\hline \multicolumn{4}{|c|}{ Mediation trough depression score* } \\
\hline Average mediated effect of & $0.079(45 \%)$ & $0.137(38 \%)$ & $0.08(16.4 \%)$ \\
\hline $\begin{array}{l}\text { CIQ sum score by current } \\
\text { depression }\end{array}$ & $95 \% \mathrm{Cl}=(-0.002,0.162), p \geqslant 0.05$ & $95 \% \mathrm{Cl}=(0.081,0.199), \mathrm{p}<0.05$ & $95 \% \mathrm{Cl}=(0.003,0.16), \mathrm{p}<0.05$ \\
\hline
\end{tabular}

Results after 2000 bootstrap replicates, * Adjusted for age, sex, smoking land cohort for GANI_MED)

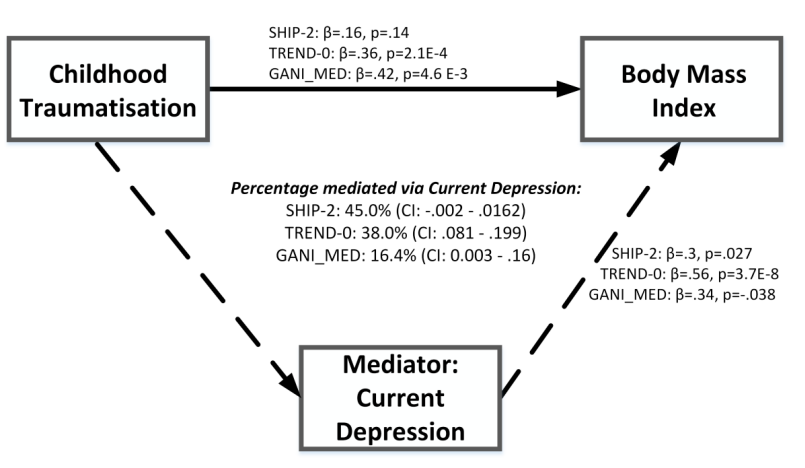

Figure 1. Direct association results between childhood trauma, current depressive symptoms and BMI. All the regression analysis are adjusted for age, sex and smoking (GANI_MED additionally adjusted for cohort).

\section{Mediation}

Results in SHIP-TREND-0 and GANI_MED indicate that the association between childhood trauma and BMI is partially mediated by symptoms of current depression. Regarding SHIP2 , we could not find significant effects, but the results pointed again in the same direction. This partial mediation makes sense as childhood trauma and depressive symptoms often co-occur and affect behaviour. Thus, they cannot be viewed as independent components. These results augment the work of another study (Francis et al. 2015), who summarized that the complex interaction between depression and body weight is not extensively understood. In a study from 2014 (McCarthyJones and McCarthy-Jones 2014), the authors could show that the association between sexual and physical abuse and the majority of physical health disorders is mediated by BMI as well as anxiety and depression. This study extends our results by amplifying the analyses on resulting physical health disorders.

\section{Limitations}

Like other studies, our analyses contain several strength and limitations: An important advantage of our analyses is the validation in three independent samples. SHIP-2 and SHIP-TREND-0 (Völzke et al. 2011) are two independent population studies selected from the same area of Germany, Western Pomerania. A difference between the two samples is the fact, that in SHIP-2, all participants are older than 30 , whereas in SHIP-TREND-0, the youngest participants are 20 years old. The difference exists since SHIP-2 is a follow-up examination with a baseline between 1997 and 2001, whereas SHIP-TREND-0 is a baseline examination. This distinction between the samples could affect the results as especially older participants might have problems to remember childhood traumatic events in retrospect. By replicating the results in three different samples of different origin (general population and clinical sample), we counteracted this limitation and thereby substantiated the results. Furthermore, childhood trauma was assessed in retrospective in adulthood. Therefore, neither the onset nor the duration of abuse or neglect could be taken into account. A further limitation is the absence of a childhood BMI value as baseline-value or potential confounder. A baseline BMI, theoretically before the abuse or neglect has happened, would have been desirable. The measurement of current depressive symptoms was conducted with the BDI-II in 
SHIP-2 and via PHQ-9 in SHIP-TREND-0 and GANI_MED. Both questionnaires measure the same construct and have an adequate correlation of Pearson's $r=0.84$ (Hawley et al. 2013). This difference in questionnaires could also be viewed as a strength as it tells us something about the generality of the findings. Thus, this discrepancy should not lead to different substantial results but it could be a reason why the results in SHIP-2 and not that strong as in the two other samples. Another crucial point is the missing of longitudinal data. In a cross sectional analysis, causality cannot be proven. However, as childhood trauma happened before the age of 16 years, we can assume that childhood trauma exhibits a causal effect on depression and BMI in adulthood.

\section{CONCLUSIONS}

The results of these analyses contribute to our understanding of the relationship between childhood trauma, depressive symptoms and bodyweight. We have emphasized that psychopathological factors like childhood trauma and depression are associated with BMI. Moreover, we could shed light on the questions, if different forms of childhood abuse play a specific role in the manifestation of BMI and if depression mediates the association between childhood trauma and BMI. Our findings confirm the importance of providing psychiatric and psychotherapeutic expertise in order to comprehend and treat the interplay of childhood trauma, depression and obesity. Consequently, clinical diagnostics are necessary in order to detect childhood maltreatment and current depressive symptoms. Especially the CTS (Glaesmer et al. 2013; Grabe et al. 2012) and PHQ-9 (Kroenke et al. 2001) represent qualified short questionnaires since both comprise reliable and valid questions, and therefore, do not burden the clinical routine. Even in clinical fields like gastroenterology, which are often confronted with the therapy of obesity, an interdisciplinary cooperation with psychotherapists is recommended to ensure a psychological diagnostic assessment, for example, by using short self-rating questionnaires like the CTS and PHQ-9.

In total, it is increasingly important to mention the psychiatric significance of obesity since obese patients with comorbid psychiatric disorders have a poor chance to decrease weight or get bariatric surgery, and in addition, have a higher risk for suicidal behaviour and suicide attempts (Wagner et al. 2013). Further studies should focus on additional psychological causes and consequences of obesity.

\section{DECLARATION OF ETHICS}

The investigations in all three studies were carried out in accordance with the Declaration of Helsinki.

\section{INFORMED CONSENT}

All participants gave their informed consent. The survey and study methods of all three studies were approved by the institutional review boards of the University of Greifswald.

\section{ACKNOWLEDGMENT/ FUNDING}

This work was supported by the German Federal Ministry of Education and Research within the framework of the e:Med research and funding concept (IntegraMent) Grant No. 01ZX1314E. The Study of Health in Pomerania is part of the Community Medicine Research net of the University of Greifswald, Germany, which is funded by the Federal Ministry of Education and Research Grant Nos. 01ZZ9603, 01ZZ0103, and 01ZZ0403; the Ministry of Cultural Affairs; and the Social Ministry of the Federal State of Mecklenburg-West Pomerania. Genome-wide data were supported by the Federal Ministry of Education, Research Grant No. 03ZIK012 and a joint grant from Siemens Healthcare, Erlangen, Germany, and the Federal State of Mecklenburg-West Pomerania. The Greifswald Approach to Individualized Medicine (GANI_MED) was funded by the Federal Ministry of Education and Research Grant No. 03IS2061A and the German Research Foundation Grant No. GR 1912/5-1.

\section{DECLARATION OF CONFLICTS OF INTEREST}

HJG has received travel grants and speakers honoraria from Fresenius Medical Care, Neuraxpharm and Janssen Cilag. He has received research funding from the German Research Foundation (DFG), the German Ministry of Education and Research (BMBF), the DAMP Foundation, Fresenius Medical Care, the EU 'Joint Programme Neurodegenerative Disorders (JPND) and the European Social Fund (ESF)'. 


\section{REFERENCES}

Bentley, T.; Widom, C. S. (2009): A 30-year follow-up of the effects of child abuse and neglect on obesity in adulthood. Obesity (Silver Spring, Md.) 17 (10), 1900-1905.

Bernstein, D.; Fink, L. (1998): Childhood trauma questionnaire (CTQ): A retrospective selfreport questionnaire and manual. San Antonio, TX: The Psychological Corporation.

Blaine, B. (2008): Does depression cause obesity?: A meta-analysis of longitudinal studies of depression and weight control. Journal of health psychology 13 (8), 1190-1197.

Bornstein, S. R.; Schuppenies, A.; Wong, M.-L.; Licinio, J. (2006): Approaching the shared biology of obesity and depression: the stress axis as the locus of gene-environment interactions. Molecular psychiatry 11 (10), 892-902.

Boynton-Jarrett, R.; Rosenberg, L.; Palmer, J. R.; Boggs, D. A.; Wise, L. A. (2012): Child and adolescent abuse in relation to obesity in adulthood: the Black Women's Health Study. Pediatrics 130 (2), 245-253.

Farmer, A.; Korszun, A.; Owen, M. J.; Craddock, N.; Jones, L.; Jones, I. et al. (2008): Medical disorders in people with recurrent depression. The British journal of psychiatry : the journal of mental science 192 (5), 351-355.

Francis, M. M.; Nikulina, V.; Widom, C. S. (2015): A Prospective Examination of the Mechanisms Linking Childhood Physical Abuse to Body Mass Index in Adulthood. Child maltreatment 20 (3), 203-213.

Glaesmer, H.; Schulz A; Häuser W; Freyberger HJ; Brähler E; Grabe HJ. (2013): [The childhood trauma screener (CTS) - development and validation of cut-off-scores for classificatory diagnostics]. Psychiatr Prax (40(4)).

Gooding, H. C.; Milliren, C.; Austin, S. B.; Sheridan, M. A.; McLaughlin, K. A. (2015): Exposure to violence in childhood is associated with higher body mass index in adolescence. Child abuse \& neglect (50), 151-158.

Grabe, H. J.; Schulz A; Schmidt CO; Appel K; Driessen M; Wingenfeld $K$ et al. (2012): [A brief instrument for the assessment of childhood abuse and neglect: the childhood trauma screener (CTS)]. Psychiatr Prax. (39(3)), 109-115.
Harrell, F. E. (2001): Regression Modeling Strategies: With Applications to Linear Models, Logistic Regression, and Survival Analysis. New York: Springer.

Hawley, C. J.; Gale, T. M.; Smith, Paul St John; Jain, S.; Farag, A.; Kondan, R. et al. (2013): Equations for converting scores between depression scales (MÅDRS, SRS, PHQ-9 and BDIII): good statistical, but weak idiographic, validity. Human psychopharmacology 28 (6), 544-551.

Herzberg, P. Y.; Goldschmidt; S (2008): Beck Depressions-Inventar (BDI-II). Revision [TBS-TK Rezension]. Report Psychologie (33(6)), 301-302.

Janowitz, D.; Schwahn, C.; Borchardt, U.; Wittfeld, K.; Schulz, A.; Barnow, S. et al. (2014): Genetic, psychosocial and clinical factors associated with hippocampal volume in the general population. Translational psychiatry 4, e465.

Kroenke, K.; Spitzer, R. L.; Williams JB (2001): The PHQ-9. Validity of a Brief Depression Severity Measure. Journal of General Internal Medicine (16 (9)), 606-613.

Mamun, A. (2007): Does Childhood Sexual Abuse Predict Young Adult's BMI A Birth Cohort Study. Obesity (15 (8)), 2103-2110.

McCarthy-Jones, S.; McCarthy-Jones, R. (2014): Body mass index and anxiety/depression as mediators of the effects of child sexual and physical abuse on physical health disorders in women. Child abuse \& neglect 38 (12), 2007-2020.

Milligan, G. W.; Cooper, M. C. (1988): A study of standardization of variables in cluster analysis. Journal of Classification (5), 181-204.

Ogden, C. L.; Carroll, M. D.; Kit, B. K.; Flegal, K. M. (2014): Prevalence of childhood and adult obesity in the United States, 2011-2012. JAMA 311 (8), 806-814.

Racine, S. E.; Wildes, J. E. (2015): Emotion dysregulation and anorexia nervosa: an exploration of the role of childhood abuse. The International journal of eating disorders 48 (1), 55-58.

Rorty, M.; Yager, J.; Rossotto, E. (1994): Childhood sexual, physical, and psychological abuse and their relationship to comorbid psychopathology in bulimia nervosa. International Journal of Eating Disorders (16 (4)), 317-34. 
Schulz, A.; Becker, M.; Van der Auwera, Sandra; Barnow, S.; Appel, K.; Mahler, J. et al. (2014): The impact of childhood trauma on depression: does resilience matter? Population-based results from the Study of Health in Pomerania. Journal of psychosomatic research 77 (2), 97-103.

Vittinghoff, E.; Glidden, D. V.; Shiboski, S. C.; McCulloch, C. E. (2012): Regression Methods in Biostatistics. Linear, Logistic, Survival, and Repeated Measures Models.

Völzke, H.; Alte, D.; Schmidt, C. O.; Radke, D.; Lorbeer, R.; Friedrich, N. et al. (2011): Cohort Profile: The Study of Health in Pomerania. International Journal of Epidemiology 40 (2), 294-307.

Völzke, H.; Schmidt, T.; Baumeister, C. O.; Schipf SE; Alte, S.; Biffar, R. et al. (2015): Prevalence trends in lifestyle-related risk factors: two cross-sectional analyses with a total of 8728 participants from the study of health in pomerania from 1997 to 2001 and 2008 to 2012. Deutsches Ärzteblatt (112), 185-190.

Wagner, B.; Klinitzke, G.; Brähler, E.; Kersting, A. (2013): Extreme obesity is associated with suicidal behavior and suicide attempts in adults: results of a population-based representative sample. Depression and anxiety 30 (10), 975-981. 